\title{
Design and Implementation of Smart Office Automation System
}

\author{
Renuka Bhuyar \\ PG student \\ Department ECE, \\ Dr. D.Y Patil School of \\ Engineering,Pune,India.
}

\author{
Saniya Ansari \\ Assistant Professor \\ Department ECE, \\ Dr. D.Y Patil School of \\ Engineering,Pune, India.
}

\begin{abstract}
Automation plays very important role in our lives. It makes the work easier and simpler so for simplified and easy living, Smart office automation system is designed in this system. This system is based on subsystems like lighting, heating. Security and alarming systems are also present. The sensors are used to extract the real time data from environment. Sensors are connected to the ARM 11 Controller. It processes the data and gives the output. Fan, bulb, buzzer are output devices connected to the controller which will work when the system crosses the threshold value. The sensor's data is continuously recorded. Fingerprint Identification module is used for security purpose. Fire alarm and emergency call is given to the service room. This data is stored in PC. This data can be viewed on other PC's through Network switch. The data can be seen on the webpage and on GUI.
\end{abstract}

\section{Keywords}

Office automation system, Security and alarming systems, emergency call.

\section{INTRODUCTION}

Nowadays most of the people spend lot of time in offices. Office environment should be leisurely so that the employees can give their best as office environment directly affects the working efficiency of employees/workers. So comfort is must and it is needed in office. In earlier decades technology at its best meant a fax machine and an electronic typewriter; today it's an iPad connected to the cloud solution. A smart office is a place that makes life easy for employees and customers, which empowers it and increases their ability to stay connected. This is achieved by making use of various advanced technology and different tools and solutions to improve the efficiency of users. As the physical boundaries are being bridged, a competitive and complex world focuses on innovation and creativity is being developed. The world is greatly experiencing the emergence of intelligent growth zones so smart office- has fast become the need of the hour.

A smart office is something that ensures the effective and optimal utilisation of IT resources and physical infrastructure. In other words, in today's generation of information technology offices are automated. There is need for transparent technological advanced environment. Thus the office automation allows the systems to become more transparent, it enables sharing of information more openly, which creates an opportunity for making an great impact across the functioning of the industrial sector and business. The use of various communication tools in the system and effective advanced automation, shows the positive impact on the growth of company or any organization and business over a period of time. The elimination of internal reporting processes, i.e. in/out timings of the workers by an open office arrangement is the advantage of smart office. The productivity can be increased through enhanced communication among team members which affects in the result. A smart office is to be designed to release full potential of employees and the workforce. It's not a miracle just innovative thinking and new technology that best fits people's needs. Office automation among other things facilitates real time communication and easy documentation.

Building sector is one of the main cause of the global energy consumption. It consumes nearly one third of total energy consumption. Smart building is like a smart home that optimizes efficiency, comfort and safety by collecting and analyzing sensor data intelligently. Modern buildings contain complex mechanical devices, sophisticated control systems and various features to improve the safety, productivity of occupants.

A smart building can be considered a super system of subsystems interconnected with building. The smart building requires connectivity between all the systems and equipments in a building. It helps building managers to visualize information and make fast and precise decisions. The smart building can be made by using various strategies. Through a building automation or building management system, the automatic centralized control of a building's different sub-systems i.e. lighting, heating, ventilation and air conditioning, and other systems are achieved. Objectives of building automation system are improved efficient operation of building systems, occupant comfort, and decrease in energy consumption and operating costs, and improve life cycle of utilities.

Building architectures consumes lot of energy. Significant amount of energy can be saved by managing the electric consumption in commercial buildings. Because of these issues, the concept of Smart office emerges. This design concept of smart office can be applied to whole building, i.e. smart office building which reduces the energy consumption. Security plays a vital role in day to day's life. The advent of fast growing technologies makes users to have high security systems. The systems should be highly secured. These identification technologies include ATM and other intelligent cards, user password based systems, and many other. But unfortunately these systems are unsecure due to hacker attacks, thefts, and forgotten passwords. RFID is also been used in security purpose. But the disadvantage of RFID is that it can be stolen or some other person can also used that ID and enter. This is not at all safe. In spite of all these shortcomings and malfunctions 
the most efficient and reliable solution for security is the biometric or fingerprint authentication based identification.

In this system, a comprehensive smart office system concentrated lighting, heating, security; alarm system is designed in order to save energy and to promote the satisfactions of the employees. With a multifaceted approach, Smart Energy Solutions achieves energy savings that capitalizes on human behaviour. By recognizing the fundamental role humans play in energy consumption, this implemented system shows the way to reduce it.

\section{RELATED WORK}

Various research papers related to office automation and smart automation systems are referred. Researchers have put focus on different controlling systems, subsystems and have controlled different parameters of smart office. Various processing parameters and results of various papers are discussed in this section.

Hang Li,In this system a comprehensive smart office system concentrated on door-access, lighting, illuminating, ventilating, heating, and reconfiguration is designed in order to save energy and promote the satisfactions of the employees[1].Catalian B. Et al, this system presents the study on how it is possible to ensure the indoor office comfort, indoor comfort is the important types of comfort (visual, acoustic, thermal, air quality, etc.) and how each of them could be analyzed. This system have discussed thermal comfort[2].Pellegrino, A. et al, This system have focused on some results that are aimed at being developing an event-driven user-centric middleware. This system have monitored and managed the energy consumption in already existing public building. The system was adopted and used in different building construction.[3]. Gupta, S.K et al, In this system a distributed solution which ensures that a consensus is attained among all occupants, irrespective of their ideal temperature preferences being in coherence or conflicting. [4].

Gaspare Boscarino et al, This system presents a simulation environment and day lighting control strategy to achieve energy-efficient lighting while it also provides desired lighting levels at the target points. The lighting strategy is based on a self-tuning multivariable controller, which maintains the illuminance levels at user-defined set-points which improves the energy consumption due to artificial lighting.[5] Kemal Akkaya et al, In this system the major challenges are to achieve occupancy monitoring in a minimally intrusive way, e.g., using the existing infrastructure in the buildings. Also it does not require the installation of any app in the users' smart devices. To develop effective data fusion techniques for improving occupancy monitoring accuracy using different sources is other challenge. This system surveys the existing works multi-modal data fusion techniques for smart commercial buildings and occupancy monitoring [6].

Shiquin Shen et al, This system proposes a novel mobility simulation framework based on behaviour patterns for office environments. Simulation time controller is the base part, on which we model the structure of offices and define behaviour patterns [7].Nati, $\mathrm{M}$ et al, This system presents the outcomes of a study that examines human interactions and mobility patterns in indoor spaces such as office environments. An initial analysis of these traces provides deeper insights into the encounter characteristics between static infrastructure devices and human-held devices. The presented findings also confirm that opportunities between mobile devices derived from observations of infrastructure devices can introduce significant errors into the modelling of such encounters [8].

Tangjian Deng et al, A smart meeting room usually refers to a working environment, which provides meeting attendees with a highly effective information acquisition and exchange space, with an aim to improve the working and decision-making efficiency. System abstracts devices spontaneous interoperations into three categories and present design and implementation of a context-aware spontaneous interoperation of information devices in building a smart meeting room [9]. Giovanni Bettinazzi et al has proposed a general method and algorithms to interconnect residential smart buildings with smart grids. Such integration system has the ability to deal with the smart building to forecast its energy consumption; the proposed method is able to learn to use of such information to forecast the energy consumption and the building occupants habits. For selecting the most appropriate appliance usage scheduling it presents a method giving a energy reduction request coming from the smart grid [10].

In the above mentioned systems parameters like Temperature (Heating, ventilating systems), light are used. Also in other papers the authors have focussed on single parameter greatly. While in this system Smoke sensor is used for fire alert and fingerprint identification module is used for security purpose which is added. 


\section{SYSTEM DESIGN}

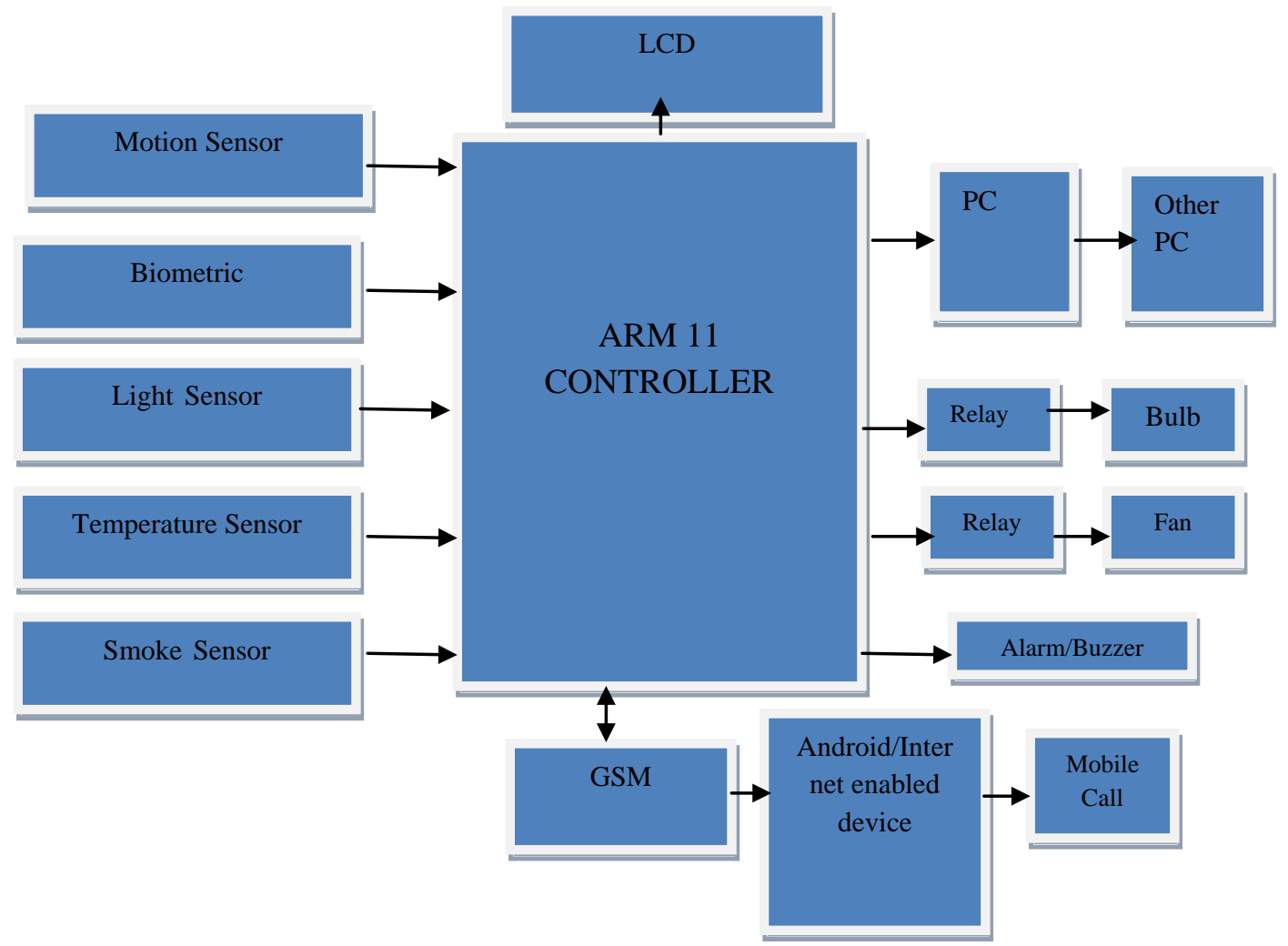

Fig.1: Block diagram of smart office system

The smart office is designed for users comfort and leisure. It works on automatic and manual mode. In automatic mode the working of system will depend On/off conditions of various sub-systems i.e. lightning and ventilating. The block diagram of smart office system is showed in Figure.1.It consists of various sensors attached to the controller.

Motion sensor will detect the presence of any person. It also counts the number of people entered in office. The system will get activate only in presence of person.PIR Motion sensor is used. Light sensor will detect the presence of light. If light intensity increases or decreases below or above some value, the bulb will glow. The system will then automatically adjust the light intensity according to the atmosphere.LDR is the sensor used. The temperature sensor will sense the surrounding temperature. If the temperature increase/decrease the fan will be $\mathrm{ON}$. LM 35 is the sensor used. Smoke sensor will detect for the presence of smoke/fire. Alarm/buzzer will get activate in presence of smoke/fire.MQ-3 is the sensor used. If there is presence of smoke/fire call will be given to the Fire extinguisher and to the service rooms that immediate service can be provided. All the sensed data is send to the ARM 11Controller.The data is displayed on LCD display.

This data sensed through different sensors is then send to computer and is stored in Office's database. This data is then send to other computers in service room from where the data is being monitored. The data can be controlled and monitor from service rooms. Other computers are connected to the main computer via Network switch. The smart office data can be remotely monitored through Android or any internet enabled device. Call will be given to the office admin if there are any changes in the parameters of office. If there is any accidental emergency, the system calls the ambulance and message is send to the service room

\subsection{Fingerprint Identification Module:}

It is used for the security purpose. It can be placed at the entry gate in office. It will contain the enrolled fingerprints of all the employees. So only the enrolled persons (whose fingerprints are enrolled) will be allowed to enter the office. If any other person tries to enter, it will show no match on LCD.

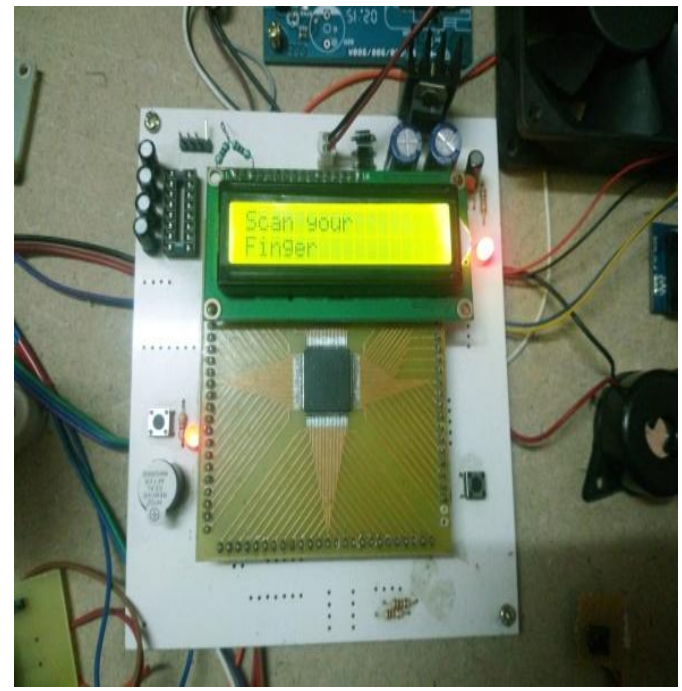

Fig.2: Fingerprint identification module used as security system 


\subsection{Alarm Security System}

In this system, if there is presence of smoke above the threshold value, the buzzer will be ON.As the buzzer will be ON, with the help of GSM Module, call will be given to the authorized user. So we will be come to know if there is an emergency. The snapshot of incoming call on authorized user is as shown in Fig.3.

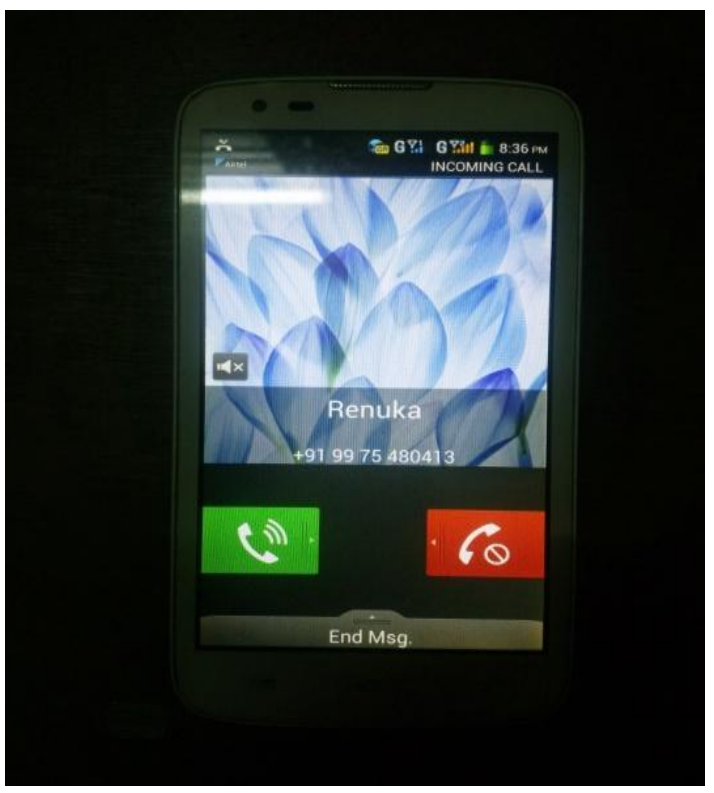

Fig.3:Showing call on authorized user.

\section{OFFICE AUTOMATION ALGORITHM}

The step by step procedure for Office automation system is showed in the Algorithm.

1) Start

2) Microcontroller Initialization

3) LCD Initialization

4) ADC Initialization

5) UART Initialization

6) IO Initialization

7) Check for Entry PIR sensor

8) If detected, display Person Detected on LCD. Make PIR flag high

9) Else display No Person on LCD. Make PIR flag Low

10) Select ADC channel 0 for temp sensor

11) Wait for $\mathrm{ADC}$ conversion complete

12) Calibrate temperature

13) Check for temp threshold

14) If threshold cross, and PIR flag is high Fan ON else OFF

15) ADC channel1 select for LDR sensor

16) Wait for $\mathrm{ADC}$ conversion complete

17) Check for light threshold
18) If threshold cross, and PIR flag is high Bulb ON else OFF

19) ADC channel2 select for humidity sensor

20) Wait for ADC conversion complete

21) Check for light threshold

22) If threshold cross, Buzzer ON else Off

23) Send all sensors data to serial port 0

24) Display it on GUI

25) Send all these values to WAMP server

26) Access this info on another Wi-Fi enabled devices

27) Repeat steps 8 to 27

\section{FLOWCHART}

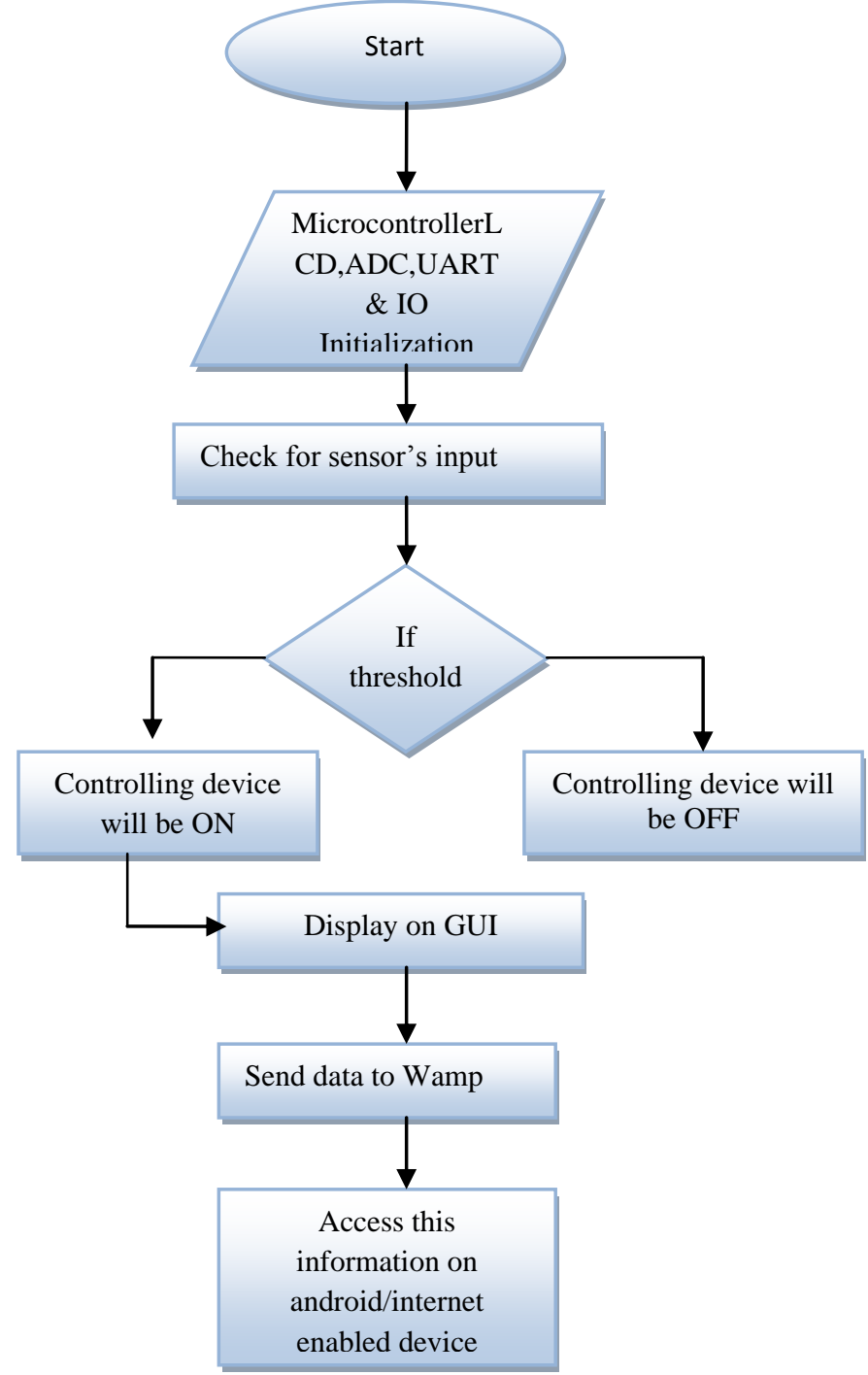

Fig.4:Flowchart of system. 


\section{RESULTS}

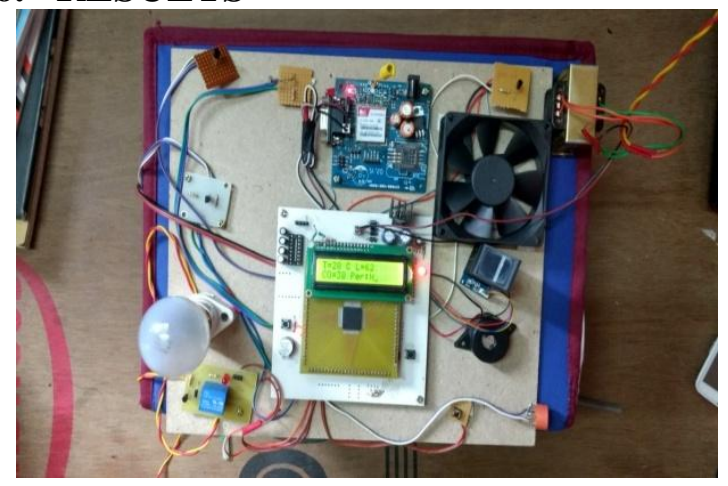

Fig.5: Hardware structure

Fig.5:shows the complete structure of hardware of proposed system. Which have used ARM lpc1769 $\mu$ c, Buzzer, relays, transformer, LCD display, Fans , LED bulbs and sensors (Temperature, , Smoke, PIR and LDR sensors).And for seeing the results of this system we need one PC and personal mobile. By combined all these elements made a new application for Smart automation for office Environment successfully. We can see the results of sensors on the LCD display and at the same time we can continuously observed the same results on Wamp server of the PC .Firstly, when give the power supply of the system, we can observe the output on the LCD screen is the name of project. Before start the micro controller, we should ON the GSM modem and wait for its connectivity. As GSM modem is connected, so we need to do ON the power supply of ARM microcontroller. After connectivity of GSM, we can see the results of all sensors on the LCD display. This shows the system result on PC as GUI(Graphical user interface).COM port have to be selected. The GUI system settings can be changed. This window has to be close before ending Eclipse software.

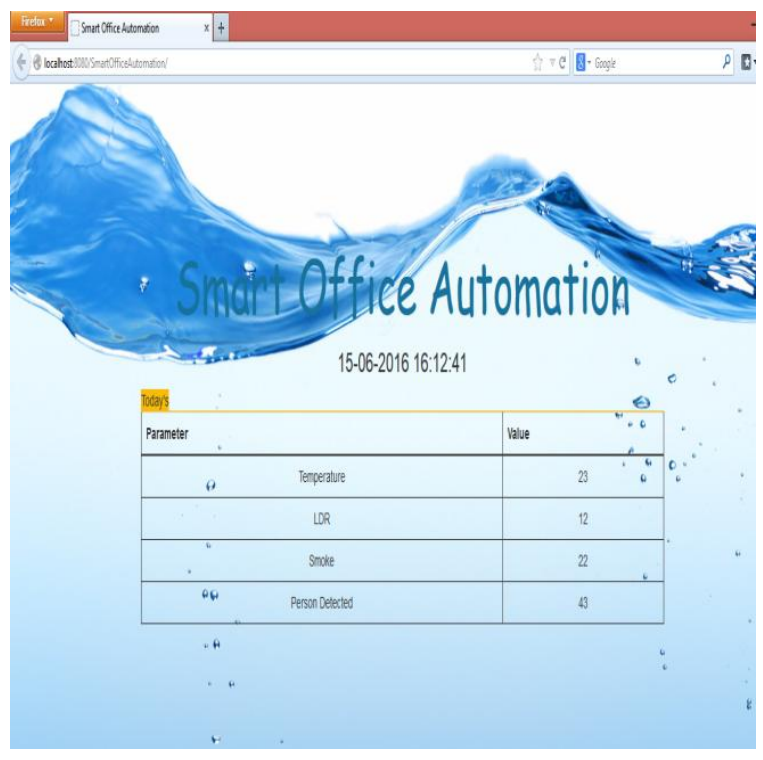

Fig.6:Snapshot of webpage.

This shows the sensors data on Webpage .It can be attached to other PC's in the service room. This data can be viewed as it is on mobile screen also. For this we have to enter the url of webpage on server.

\section{CONCLUSION}

The illuminating, lighting, heating, ventilating ,door access, smoke detection systems are being designed. Fingerprint biometric is used for security purpose. Other person cannot enter the office area. Fire alarm system is used. Whenever the threshold is crossed, Alarm will be $\mathrm{ON}$ and call will be given on mobile in service room. The smart office system in the system is based on an independent smart office and then expanded to the whole smart building. In this smart office system, two working modes automatic mode and manual mode are use. The manual mode is viewed as a supplement of the automatic mode.

\section{ACKNOWLEGEMENT}

This study is useful for the smart automation systems for office environment and in understanding the different subsystems for office use by using ARM controller and it is used to reduce the power consumption. It is also useful in studying fully automated systems in office and industrial sector.

\section{FUTURE SCOPE}

This system of smart office can be extended to whole building. Energy consumption can be reduced to great extent if this system is applied to whole building. As the smart office is not only a product of modern technology, but also a product that can save energy. This system can be helpful to energy saving and employee's satisfactions. Comparisons between the real office environment power consumption and smart office environment power consumption should be made. And then comparisons should be made under different methods.

\section{REFERENCES}

[1] Hang Li;Inst of Autom.\& Inf.Sys., Tech. Univ. Munchen, Garching, Germany"A novel design for a comprehensive smart automation system for the office environment" Emerging Technology and Factory Automation (ETFA), 2014 IEEE

[2] Catalin Bujdei and Sorin Aurel ,Moraru Brasov, Romania "Ensuring Comfort in Office Buildings: Designing a KNX Monitoring and Control System" Intelligent Environments (IE), 2011 7th International Conference IEEE,2011

[3] Anna Pellegrino, Valerio R.M. Lo Verso, Laura Blaso "Lighting control and monitoring for energy efficiency: a case study focused on the interoperability of building management systems" Industry Applications, IEEE Transactions on (Volume:PP, Issue: 99 )

[4] Gupta, S.K., Mishra, S.,Wen, J.T. "Collaborative Energy and Thermal Comfort Management Through Distributed Consensus Algorithms"Automation Science and Engineering, IEEE Transactions on (Volume:12, Issue: 4 ) 2015

[5] Gaspare Boscarino, Mehrdad Moallem "Daylighting Control and Simulation for LED-Based EnergyEfficient Lighting Systems" IEEE Transactions on Industrial Informatics (Volume:12, Issue: 1 )2015

[6] Kemal Akkaya et al "IoT-based Occupancy Monitoring Techniques for Energy-Efficient Smart Buildings" IEEE 2015 
[7] Shiquin Shen,Nanjing,Ningning Cheng,Guihai Chen "A Behvior Pettern Based Mobility Simulation Framwork for Office Environments"Wireless Communications and Networking Conference,2009WCNC 2009,IEEE

[8] Gluhak, A. ; Martelli, F. ; Verdone, R. "Measuring and Understanding Opportunistic Co-presence Patterns in Smart Office Spaces" Green Computing and Communications (GreenCom), 2013 IEEE and Internet of Things (iThings/CPSCom), IEEE International Conference on and IEEE Cyber, Physical and Social Computing
[9] Tangjian Deng, Feng, L. ; Yue Suo ; Yu Chen "Spontaneous Interoperation of Information Appliances in a Smart Meeting Room" Intelligent Systems and Applications (ISA), 2010 2nd International Workshop

[10] Donatella, Sciuto;Politec di Milano,Milan,Italy;Alessandro Antonio Nacci "On how to design smart energy-efficient buildings" Embedded and Ubiquitous Computing(EUC),2014 $12^{\text {th }}$ IEEE International Conference. 